\title{
Do speakers produce discourse connectives rationally?
}

\author{
Frances Yung $^{1}$ and Vera Demberg ${ }^{1,2}$ \\ ${ }^{1}$ Dept. of Language Science and Technology \\ ${ }^{2}$ Dept. of Mathematics and Computer Science, Saarland University \\ Saarland Informatic Campus, 66123 Saarbrücken, Germany \\ \{frances, vera\}@coli.uni-saarland.de
}

\begin{abstract}
A number of different discourse connectives can be used to mark the same discourse relation, but it is unclear what factors affect connective choice. One recent account is the Rational Speech Acts theory, which predicts that speakers try to maximize the informativeness of an utterance such that the listener can interpret the intended meaning correctly. Existing prior work uses referential language games to test the rational account of speakers' production of concrete meanings, such as identification of objects within a picture. Building on the same paradigm, we design a novel Discourse Continuation Game to investigate speakers' production of abstract discourse relations. Experimental results reveal that speakers significantly prefer a more informative connective, in line with predictions of the RSA model.
\end{abstract}

\section{Introduction}

Discourse relations connect units of texts to a coherent and meaningful structure. Discourse connectives (DC), e.g., but and so, are used to signal discourse relations. In Example (1), the connective as is used to mark the causal relation between the two clauses.

(1) That tennis player has been losing his matches, as we know he is still recovering from the injury.

However, discourse relations can often be expressed by more than one DC, or not be marked by an explicit connective at all (these are referred to as implicit relations). For example, the connectives since or because can alternatively be used in Example (1). Note however that there can be small differences in meaning between alternative connectives: because stresses more strongly that the reason is the new information in the discourse.

There is a large body of literature on the comprehension of DCs and unmarked discourse relations (see for example Sanders and Noordman (2000)), but the production of discourse relations is under-studied. Patterson and Kehler (2013) and Asr and Demberg (2015) investigate the choice of using a DC vs. omitting it, and find that explicit connectives are more often used when the discourse relation cannot be easily predicted from prior context. More recently, Yung et al. (2017, 2016) proposed a broad-coverage RSA model to account for relation signaling, and showed that the RSA-based modeling improves the prediction of whether a relation is marked explicitly or not.

Nonetheless, it is still unclear, what factors affect the speaker's choice of a specific explicit connective. Given the previous success of the RSA account in predicting connective presence in a corpus, we here set out to investigate whether the choice of DCs follows the game-theoretic Bayesian model of pragmatic reasoning (Frank and Goodman, 2012). As broad-coverage corpus analyses can be very noisy and can include a lot of confounding effects, in particular with respect to small meaning differences between connectives, which we cannot control in a corpus study, we here test for an RSA effect in a tightly controlled experimental setting.

\section{Background: The rational account of linguistic variation}

Natural language allows us to formulate the same message in many different ways. The rational speech act (RSA) model (Frank and Goodman, 2012; Frank et al., 2016) explains linguistic variation in terms of speakers' pragmatic reasoning 
about the listeners' interpretation in context. Using Bayesian inference, the model formalizes the utility of an utterance to convey the intended meaning in context $c$. In our case, the utterance is a $D C$ and the meaning is a discourse relation $r$. Utility is defined in Equation 1:

$$
\begin{aligned}
& \operatorname{Utility}(D C ; r, c) \\
& =-\log P(r \mid D C, c)-\operatorname{cost}(D C)
\end{aligned}
$$

$-\log P(r \mid D C, c)$ quantifies the informativeness of $D C$, i.e. how likely the intended meaning $r$ can be interpreted by the listener in context $c$. $\operatorname{cost}(D C)$ quantifies the production cost of the utterance. The probability that a rational speaker chooses $D C$ is proportional to its utility.

$$
P(D C \mid r, c) \propto \exp ^{\alpha U t i l i t y(D C ; r, c)}
$$

According to the RSA theory, the rational utterance should provide the most unambiguous information for the listener, and, at the same time, be as brief as possible. These goals correspond to Grice's Maxims of effective communication (Grice, 1975).

The RSA model has been shown to account for speakers' choice during production for various phenomena, such as referential expressions (Degen et al., 2013; Frank et al., 2016), scalar implicatures (Goodman and Stuhlmüller, 2013), yes-no questions (Hawkins et al., 2015), shape descriptions (Hawkins et al., 2017) and uncertainty expressions (Herbstritt and Franke, 2017). In these existing works, speakers' utterances are collected by experiments in the form of referential language games. Although various types of speaker utterances have been investigated, the intended meanings to be conveyed in the experiments are commonly the identification of concrete, visible objects or attributes, such as figures, colors and quantities presented in pictures.

\section{Methodology}

In this work, we conduct language game experiments to test the rational account of speakers' production of discourse relations. In contrast to previous approaches that use RSA to predict the presence or absence of DCs in corpus data (Yung et al., 2016, 2017), we compare the theoretical choice of RSA with the choice of human subjects. To our knowledge, this is the first attempt to manipulate the production of abstract meanings in the language game paradigm.
According to RSA, among alternative DCs that are literally correct for a given intended discourse relation, speakers prefer the DC with larger $P(D C \mid r, c)$ and thus larger utility (Equation 2). Since DCs are generally frequent expressions consisting of no more than a few words, we assume that the production cost for all DCs is constant. Therefore, the DC that is more informative in context (larger $P(r \mid D C, c)$ ) is the one preferred by the speaker (Equation 1).

We use crowdsourcing to collect discourse processing responses from naive subjects, following previous success (Rhode et al., 2016; Scholman and Demberg, 2017). It is, nonetheless, challenging to manipulate the intended meaning in a production scenario, because discourse relation cannot be presented visually, as in other referential language games. We design a novel Discourse Continuation Game that induces the subjects to choose a DC, among multiple options with different levels of informativeness, to convey a particular discourse relation.

\subsection{Task and stimulus design}

In each Discourse Continuation Game, the subject is asked to choose a DC as a hint for another player, Player 2, who is supposed to guess how the discourse will continue ${ }^{1}$. There are three possible continuations and three DC options in each question. The subject (Player 1) is told that both players see the possible continuations but only Player 1 knows which continuation is the target. Figure 1 shows the screen shot of one of the questions.

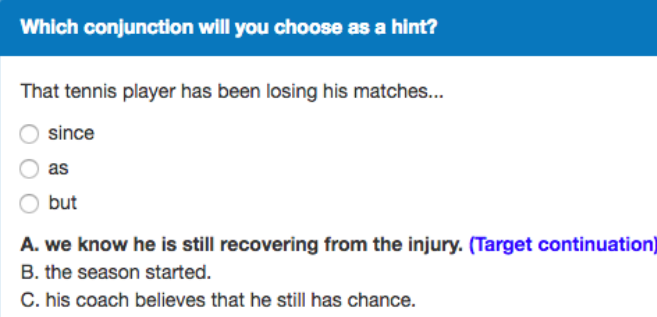

Figure 1: Sample question of the Discourse Continuation Game, under the with competitor condition. Continuation $B$ is replaced by "he was close in every match." under the no competitor condition.

\footnotetext{
${ }^{1}$ We focus on speaker's production in this work, so the listener, Player 2, does not exist. Fake responses are generated by the system during the experiment. See Section 3.2.
} 
Each continuation option represents a discourse relation and the target continuation is the discourse relation we want the subjects to produce. For the example in Figure 1, continuations $A, B$ and $C$ represent causal, temporal and concession relations respectively.

The three DC options differ in the level of informativeness in context, i.e. $P(r \mid D C, c)$. For the example in Figure 1, since is the ambiguous $D C$ because it can be used to mark the target continuation $A$ (causal relation), as well as continuation $B$ (temporal relation). As is the unambiguous DC because, among the available continuations, it can be used to mark the target continuation only. But is the unrelated DC because it is used to mark continuation $C$, which is not the target.

When the speaker utters since, continuation $B$ can be seen as the competitor of the target continuation $A$. We modify the informativeness of since by replacing the competitor continuation with another unrelated continuation. Under this no competitor condition, both since and as are unambiguous $D C$ s for the target continuation $A$. The no competitor condition serves as the control condition because DC choice of a particular utterance can be subject to other factors on top of informativeness. By keeping the target identical and only manipulating the set of alternative continuations, we can control for fine nuances in connective meaning: if a connective is more suitable for marking the target continuation than another one, this will be the same for both conditions.

\begin{tabular}{lll|l}
\hline$D C$ & & context $c$ & $P(r \mid D C, c)$ \\
\hline ambiguous & since & with comp. & lower \\
unambiguous & as & with comp. & high \\
unrelated & but & with comp. & lowest \\
\hline ambiguous & since & no comp. & high \\
unambiguous & as & no comp. & high \\
unrelated & but & no comp. & lowest \\
\hline
\end{tabular}

Table 1: Level of informativeness of the DC options in the Discourse Continuation Game example in Figure 1.

The level of informativeness of various DC options for target continuation $A$ is summarized in Table 1 . When the speaker intends to convey the discourse relation represented by continuation $A$, both since and as are literally correct DCs, so both DCs are similarly likely to be selected under the no competitor condition. But, according to RSA theory, the unambiguous DC as is pragmatically preferred when there is a competitor in context. We crowdsource responses of the Discourse Continuation Game to evaluate this RSA prediction.

\subsection{Experiment}

We constructed 36 stimuli similar to the example in Figure 1, covering eight ambiguous DCs, as shown in Table 2.

\begin{tabular}{lll|l}
\hline $\begin{array}{l}\text { ambiguous } \\
\text { connective }\end{array}$ & $\begin{array}{l}\text { unambiguous } \\
\text { alternative }\end{array}$ & $\begin{array}{l}\text { stimulus } \\
\text { count }\end{array}$ & total \\
\hline \hline and & $\begin{array}{l}\text { also } \\
\text { and then } \\
\text { therefore }\end{array}$ & 2 & \\
& so & 1 & \\
& at the same time & 4 & 10 \\
\hline while & but & 1 & \\
& when & 1 & \\
& however & 1 & 7 \\
\hline as & since & 2 & \\
& while & 1 & \\
& whilst & 1 & 4 \\
\hline or & otherwise & 3 & \\
& alternatively & 1 & 4 \\
\hline meanwhile & however & 1 & 1 \\
since & as & 5 & 5 \\
then & after that & 1 & 1 \\
when & if & 4 & 4 \\
\hline & & & 36 \\
\hline
\end{tabular}

Table 2: List of DCs covered in the stimuli

Since many readings are possible if a discourse relation is unmarked, to make sure that the stimuli are valid, we conduct pretests by recruiting a separated group of participants to fill in any words that connect the first sentence with the continuation options. A stimulus is excluded or revised if, for any of the 3 continuation options, any pretest participant fills in a DC that is among the 3 DC options but is not the matching DC (or one of the matching DCs for continuation $A$ ). The pretest makes sure that: 1) all options are compatible with the intended literal DC; 2) the target continuation is compatible with both of the DCs that match it; and 3 ) continuation $B$ and $C$ are not compatible with the DCs which are not their literal connectives in the experiment.

The 36 stimuli (each in two conditions) were divided up into 12 separate lists, each containing 6 items. Each participant saw 3 items in each of the two conditions. An additional 6 filler items were added to each of the lists, resulting in a total of 12 different questions in a list. The order of items in a list was randomized. For each of the 12 lists, we collected 20 responses, resulting in a total 
of 240 native-English-speaking participants who took part in the experiment. 127 participants are females and 73 are males. Their average age is 34 . 148 participants come from the United Kingdom, 34 from the United States and 18 from other countries, including Canada, Ireland etc. The participants were recruited through the Prolific platform. They took on average 8 minutes to complete the task, and were reimbursed for their efforts with $0.8 \mathrm{GBP}$ each. The filler questions had the same form as the stimuli, except that continuations $B$ or $C$ were set as the target instead of the experimentally interesting continuation $A$. Responses from participants who chose more than 6 non-matching DCs in their list were excluded and recollected. The experimental interface was constructed using Lingoturk (Pusse et al., 2016).

The experimental interface was designed to resemble a communication scenario where two players interact at real time, although the responses of "Player 2" were actually automatically generated by the system, and were shown to the subject with a time lag of 4 seconds. "Player 2" was programmed to be an rational Gricean pragmatic listener, who in the unambiguous condition always chose the continuation that best fits the connective, and who supposed that the speaker would choose an unambiguous DC when there was a competitor in context. For example, if the participant chose the ambiguous since, "Player 2" would guess continuation $B$, assuming that the participant would have chosen the unambiguous $a s$ if he meant continuation $A$.

To motivate the participants, they were rewarded with a bonus of 0.06 GBP for each question where the "Player 2" successfully guessed the target continuation.

\section{Results}

We calculate the agreement among the participants for each stimulus by

$$
\frac{\text { Count(majority response) }}{\text { Count(all response) }}
$$

and average it over the items. The average agreement of the filler items is $87 \%$ while that of the stimulus items is $68 \%$ and $71 \%$ respectively for the no- and with competitor conditions. The agreement of the filler items is higher than that of the stimulus items. It is expected because only one of the three connective options literally matches the target continuation in the filler items while two of the options are literally correct in the stimulus items. The agreement under the no competitor condition is slightly lower than the with competitor condition. This follows our prediction that, under the no competitor condition, participants more freely choose between the two literally correct options, because they are equally informative.

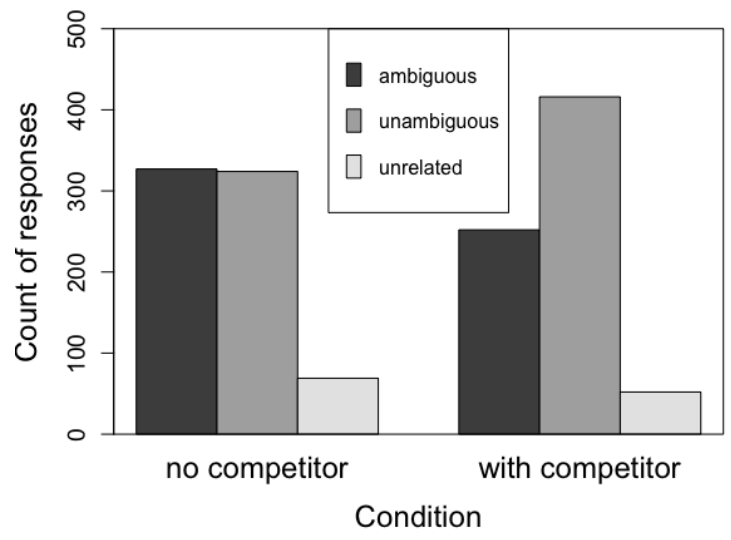

Figure 2: Distribution of participant responses.

The distribution of the participant responses is shown in Figure 2. In both conditions, most of participants choose one of the connectives that fits the target relation (i.e., the ambiguous or unambiguous DC). This shows that our stimuli are valid, because both options are literally correct for the target continuation.

Also, the results show that the distribution of connective choice differs between the two conditions: In the no competitor condition, where both the ambiguous and unambiguous DCs are similarly informative, speakers' choice between the two options is evenly distributed. In the condition with the competitor, the ambiguous connective is chosen significantly less often than in the no-competitor condition. This is the expected effect according to the RSA model, as the ambiguous connective is less informative in the condition with the competitor.

Moreover, we are also interested to see if there is a learning effect as the trials progress. When the subjects chose an unambiguous connective, a positive feedback was displayed to the subjects saying that Player 2 correctly guessed the continuation. Figure 3 shows the distribution of subject responses grouped by the number of correct an- 


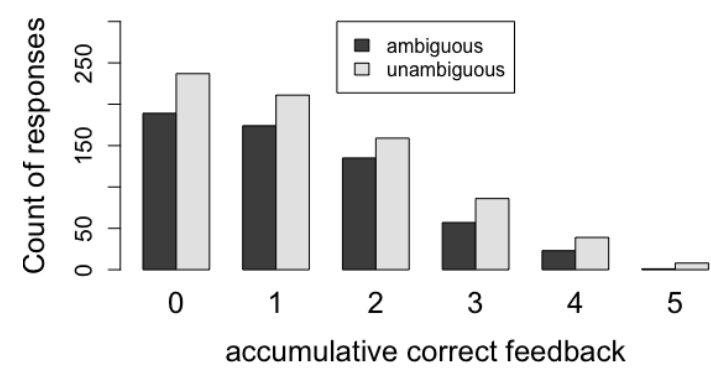

Figure 3: Distribution of participant responses by the number of previous positive feedbacks excluding the fillers

swers they previously got, excluding the fillers. Increased preference for unambiguous connectives is not observable; the subjects prefer an unambiguous connective since the first question and the tendency persists until the end of the trial.

We test for significance of the effects of the with/no competitor conditions as well as previous positive feedbacks on connective choice using a logistic mixed effects model. Responses choosing the unrelated DCs are not taken into account. We included by-subject and by-stimulus random intercepts, as well as random slopes for the effect of the condition under both subject and item. The regression values of the effects are reported in Table 3. Statistical analyses were performed using the $l m e 4$ package (Bates et al., 2015), version 1.115 . The with competitor condition was confirmed to have statistically significant positive effect on the choice of unambiguous DC, but no significant effect from the number of previous positive feedbacks is detected. Further investigation is necessary to evaluate the effect of pragmatic feedbacks, possibly in longer trials of experiment.

To summarize, speakers do not have a preference choosing either of the DC options that are literally appropriate for the target discourse relation when both DCs are similarly informative. However, when one of the literal DCs is ambiguous in context, the speaker chooses the unambiguous one to facilitate listener's comprehension. These results support the prediction of the RSA theory.

\section{Conclusion}

This work investigates the preference of speakers' production of DCs for an intended discourse rela-

\begin{tabular}{lllll}
\multicolumn{2}{l}{ Fixed effects: } & & & \\
\hline & $\beta$ & SE & $t$ & $p$ \\
intercept & -.0891 & .272 & -.328 & .743 \\
with comp. & .649 & .177 & 3.676 & $.000237^{* * *}$ \\
feedback & .0679 & .0634 & 1.072 & .284 \\
\hline Random effects: & & & \\
Groups & Name & Variance & SD & Corr. \\
subject & intercept & .186 & .431 & \\
& wth comp. & .117 & .342 & -1.00 \\
stimuli & intercept & 2.047 & 1.431 & \\
& wth comp. & .458 & .677 & -.65 \\
\hline
\end{tabular}

Table 3: The regression values of the logistic mixed effect model.

tion. According to the responses of subjects participating in a specially designed Discourse Continuation Game, we found that speakers prefer a more informative, less ambiguous DC when it is necessary for effective communication. The results are consistent with predictions of the RSA model, showing that speakers choose their utterance by pragmatic reasoning when planning the production of abstract meanings, such as discourse relations. The results are also consistent with the earlier broad-coverage model by Yung et al. (2017), that speakers prefer to explicitly mark discourse relations when they predict that the relation is hard to interpret if it is unmarked.

The Discourse Continuation Game successfully extends the referential language game paradigm to test the production of abstract, non-visible meanings. A limitation of the current first study is that the alternative completions of the sentence are provided explicitly to the speaker and the comprehender, which is not the case in natural communication. Therefore, the current study only provides information on what humans can do, but not yet necessarily on what they usually do in natural communication. We plan to extend our work to more realistic settings in subsequent work.

\section{Acknowledgments}

This research was funded by the German Research Foundation (DFG) as part of SFB 1102 Information Density and Linguistic Encoding and the Cluster of Excellence (MMCI).

\section{References}

Fatemeh Torabi Asr and Vera Demberg. 2015. Uniform information density at the level of discourse relations: Negation markers and discourse connec- 
tive omission. Proc. of the International Conference on Computational Semantics.

Douglas Bates, Martin Mächler, Ben Bolker, and Steve Walker. 2015. Fitting linear mixed-effects models using lme4. Journal of Statistical Software, 67(1):1-48.

Judith Degen, Michael Franke, and Gerhard Jager. 2013. Cost-based pragmatic inference about referential expressions. In Proceedings of the annual meeting of the cognitive science society, volume 35.

Michael C Frank, Andrés Gómez Emilsson, Benjamin Peloquin, Noah D Goodman, and Christopher Potts. 2016. Rational speech act models of pragmatic reasoning in reference games.

Michael C Frank and Noah D Goodman. 2012. Predicting pragmatic reasoning in language games. Science, 336(6084):998-998.

Noah D Goodman and Andreas Stuhlmüller. 2013. Knowledge and implicature: Modeling language understanding as social cognition. Topics in cognitive science, 5(1):173-184.

H Paul Grice. 1975. Logic and conversation. Syntax and Semantics, 3:41-58.

Robert XD Hawkins, Michael C Frank, and Noah D Goodman. 2017. Convention-formation in iterated reference games. In Proceedings of the 39th Annual Conference of the Cognitive Science Society. Cognitive Science Society.

Robert XD Hawkins, Andreas Stuhlmüller, Judith Degen, and Noah D Goodman. 2015. Why do you ask? good questions provoke informative answers. In $\mathrm{CogSci}$. Citeseer.

Michele Herbstritt and Michael Franke. 2017. Modeling transfer of high-order uncertain information. CogSci.

Gary Patterson and Andrew Kehler. 2013. Predicting the presence of discourse connectives. Proc. of the Conference on Empirical Methods in Natural Language Processing, pages 914-923.

Florian Pusse, Asad Sayeed, and Vera Demberg. 2016. Lingoturk: Managing crowdsourced tasks for psycholinguistics. Proc. of the North American Chapter of the Association for Computational Linguistics.

H Rhode, A. Dickinson, N. Schneider, C. N. L. Clark, A. Louis, and B. Webber. 2016. Filling in the blanks in understanding discourse adverbials: Consistency, conflict, and context-dependence in a crowdsourced elicitation task. Proc. of the Linguistic Annotation Workshop.

Ted JM Sanders and Leo GM Noordman. 2000. The role of coherence relations and their linguistic markers in text processing. Discourse processes, 29(1):37-60.
Merel Cleo Johanna Scholman and Vera Demberg. 2017. Examples and specifications that prove a point: Identifying elaborative and argumentative discourse relations. Dialogue \& Discourse, 8(2):56-83.

Frances Yung, Kevin Duh, Taku Komura, and Yuji Matsumoto. 2016. Modeling the usage of discourse connectives as rational speech acts. Proc. of the SIGNLL Conference on Computational Natural Language Learning, page 302.

Frances Yung, Kevin Duh, Taku Komura, and Yuji Matsumoto. 2017. A psycholinguistic model for the marking of discourse relations. Dialogue \& Discourse, 8(1):106-131. 


\section{A Stimuli and fillers of the experiment}

Continuations $A, B_{w t h}$ and $C$ are displayed to the subjects under the with competitor condition, as well as in the fillers. Continuations $A, B_{n o}$ and $C$ are displayed under the no competitor condition. Continuation $A$ is set as the target in the stimulus questions, while continuations $B_{w t h}$ or $C$ are the targets in the fillers. The connective options are in the order: ambiguous / unambiguous / unrelated.

1 Hard work is the key to success...

[ and / also / unless ]

$A$. patience is important.

$B_{w t h .} \quad$ honesty is the key to friendship.

$B_{n o .} \quad$ you are always lucky.

$C$. you are a genius.

2 Harry was born in Scotland...

[ and / and then / but ]

$A$. he lived in Glasgow for 20 years.

$B_{w t h .} \quad$ his ancestors had origined from Scotland.

$B_{\text {no. }} \quad$ both his parents are not Scottish.

$C$. he would not have said so.

3 I listened to music on my mobile phone...

[ while / when / because ]

$A$ I I was walking back home from work.

$B_{w t h .} \quad$ I knew there are more important things I should do instead.

$B_{n o .} \quad$ it helped me to concentrate.

$C$ I I was bored waiting for you for half an hour.

4 I will buy a bag for my son as promised...

[ or / otherwise / because ]

A. $\quad$ he will be very disappointed.

$B_{w t h .} \quad$ I will buy him a watch instead.

$B_{n o .} \quad$ he did well in his exams.

$C$. it is his birthday tomorrow.

5 You must have been studying this afternoon...

[ since / as / but ]

$A$ I I did not hear music from your room.

$B_{w t h .} \quad$ you came back from school.

$B_{n o .} \quad$ it doesn't mean you will certainly get good marks in the exam.

$C$. John has been playing video games all the time.

6 I had been longing for a cup of coffee...

[ since / as / so ]

A.

you woke me up at five this morning.

$B_{w t h .} \quad$ the teacher of the first class came in.

$B_{n o .} \quad$ I rushed to the cafeteria as soon as the bell rang.

$C$. please do me a favour and buy me an espresso.

7 I will finish this homework now...

[ then / after that / although ]

A.

$B_{w t h}$

I will go to chill with my friends.

$B_{n o}$.

I can have something to hand in tomorrow.

C.

I don't know the answers for half of the questions.

it is not interesting at all. 
8 Big cities are fun to visit ... [ and / therefore / but ]

$A$.

$B_{w t h .}$

$B_{n o}$.

C.
I visit at least one of those every year. they are usually easier to access as well. surprisingly my sister prefers small towns. unfortunately those places are often packed with tourists.

9 Your joints will feel better... [ when / if / but ]

$A$.

$B_{w t h}$

$B_{\text {no. }}$

$C$. you do these stretches regularly.

the summer comes.

still you should not start running yet.

the symptom will never go away unfortunately.

10 The older children stopped talking at once...

[ as / since / but ]

A.

they understood that it was not a joke.

$B_{w t h}$ the train approached the station.

$B_{\text {no. }}$ that lasted for a minute only.

C. the younger ones were still noisy.

11 Jane finished the obstacle course the fastest...

[ and / so / but ]

$A$.

$B_{w t h}$

$B_{n o}$.

$C$. she ended up winning the first prize overall.

Mary finished it very quickly, too.

she was disqualified.

still she could not win.

12 I started to watch over my calorie intake...

[ since / as / so ]

A. you said I ate too much.

$B_{w t h .}$ I moved back to my parents'.

$B_{\text {no. }}$ I might finally be able to lose some weight.

$C$. you'd better not offer me chocolates and chips.

13 Let's just follow Peter's idea... [ or / otherwise / because ]

A.

$B_{w t h .}$

$B_{\text {no. }}$

$C$. we will never finish the project on time.

we can adopt Tom's alternative instead.

no one is suggesting anything better.

I think his idea is simple but great.

14 Maggie grabbed her coat and sweater...

[ as / while / but ]

A.

$B_{w t h}$

$B_{\text {no. }}$

C. she followed the crowd into the playground.

it was snowing outside.

Tom went out with short sleeves.

she did not take her hat.

15 Mark was almost an hour late to the station last evening...

[ while / but / and ]

A.

$B_{w t h}$

$B_{\text {no. }}$

$C$.
Harry was even two hours late. he was on his way to London. he even said he was going to quit. he was late again this morning. 
16 Dave ordered a tall glass of fine scotch...

[ as / since / but ]

A.

we could order what ever we want.

$B_{w t h .}$

the host was giving a speech.

$B_{n o}$.

he could not finish half of it.

$C$. Mary just ordered a soft drink.

17 Mary always wore a fancy dress to a ball...

[ when / if / whereas ]

$A$.

her boyfriend was going as well.

$B_{w t h .}$

she was at her $20 \mathrm{~s}$.

$B_{\text {no. }}$

she did not care much about her hair.

$C$.

she dressed casually to work.

18 That pizzaria has always been my favourite...

[ since / as / but ]

A.

I like Italian food a lot.

$B_{w t h .}$

I had dinner with Jill there two years ago.

$B_{\text {no. }}$

my boyfriend doesn't really like it.

$C$.

I think this restaurant is not bad, too.

19 My parents will visit Canada again in December...

[ and / and then / although ]

$A$.

$B_{w t h}$

$B_{\text {no. }}$

$C$.

20 I am sure David will burst into tears... [ when / if / but ]

A.

$B_{w t h .}$

$B_{n o}$

$C$.

21 Leo is taking orders from the guests...

[ while / at the same time / so ]

A.

$B_{w t h}$

$B_{\text {no. }}$

$C$.

22 Peter was watching the baseball match on TV this morning...

[ while / at the same time / because ]

A.

$B_{w t h .}$

$B_{\text {no. }}$

$C$.

23 Please buy some fruits for me...

[ and / and then / if ]

A.

$B_{w t h .}$

$B_{\text {no. }}$

$C$.

don't forget the milk.

you still have money left.

you pass by a supermarket. they will visit South America in spring.

it will be their thrid visit in two years.

the air tickets are expensive in that season.

they hate cold weather.

his children come to visit one day.

he comes home tonight.

Kathy probably will not react much.

that will be tears of happiness.

George is serving the food.

there are too many tables for him to serve alone.

he is not able to pick up the call right now.

have patience, he will come to our table sooner or later.

his wife was making breakfast for him in the kitchen.

he didn't understand the rules at all.

there were not any other good shows on TV.

he recently became a fan of the team that was playing.

come home immediately afterwards. 
24 Sam is going on a business trip to Seoul....

[ while / at the same time / and ]

$A$.

$B_{w t h .}$

$B_{n o}$.

$C$.

25 That task took me a lot of time... [ and / so / but ]

A.

$B_{w t h .}$

$B_{n o}$

$C$. his children are going to a summer camp.

he is not very optimistic about the Korean market. he will come back with signed contracts.

later he will travel to Japan for an exhibition.

I expected a higher reward.

it was so boring.

it was not the worst.

I enjoyed doing it.

26 The carnival was held on the main street for a week...

[ while / at the meantime / because ]

A.

a film festival was being held in the same period.

$B_{w t h .}$

it was held in the park for only one day.

$B_{\text {no. }}$

the central park was not big enough.

$C$.

people complained that three days were too short.

27 The cat always behaves weird at night ...

[ when / if / but ]

$A$. we have a visitor at home.

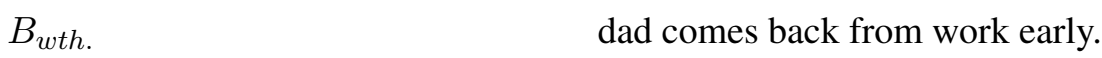

$B_{\text {no. }} \quad$ she was normal last night.

$C$. $\quad$ she will be fine the next morning.

28 The cleaning lady will come to clean our house in the morning... [ and / also / otherwise ]

A.

she will wash the cars.

$B_{w t h .}$

we can just leave the dishes in the kitchen.

$B_{n o}$ we will have to do it ourselves.

$C$. I think the house will just be in a mess forever.

29 The current situation is likely to change... [ while / however / before ]

A.

$B_{w t h}$

$B_{\text {no. }}$

C.

30 The talk will be delayed for an hour... [ meanwhile / however / because ]

$A$.

$B_{w t h}$

$B_{\text {no. }}$

$C$.

31 The teddy bear dropped from the baby's hand...

[ and / and then / as ]

A.

$B_{w t h .}$

$B_{\text {no. }}$

C. our standard of living is unlikely to improve. the management is planning the next move. the summer holiday starts.

you even notice it.

the conference room is already full of people.

people are having a coffee break.

there is a technical problem.

the speaker is coming late.

he cried aloud.

he has dropped it twice in a minute.

he fell asleep.

the stroller entered the elevator. 
32 That tennis player has been losing his matches...

[ since / as / but ]

$A$.

$B_{w t h .}$

we know he is still recovering from the injury.

$B_{n o}$.

the season started.

$C$.

he was close in every match.

his coach believes that he still has chance.

33 The next concert will be held this summer here in this city... [ and / so / but ]

$A$.

$B_{w t h .}$

$B_{n o .}$

$C$.

34 We fell asleep immediately... [ as / whilst / but ]

A.

$B_{w t h .}$

$B_{\text {no. }}$

$C$. we are definitely going.

I heard that it will be an outdoor concert.

unfortunately I cannot go this time.

the dates are not yet confirmed.

the moon rose higher in the sky.

we had been working the whole day.

we woke up shortly in the middle of the night.

the kids stayed up until early in the morning..

35 We should not walk but take the bus...

[ or / alternatively / although ]

A.

$B_{w t h .}$

$B_{\text {no. }}$

$C$. we can take a taxi instead.

we will not arrive on time.

it would have been nice to walk through the forest.

it is still not the fastest way to get there.

36 You should bring something to eat...

[ or / otherwise / although ]

A.

$B_{w t h .}$

$B_{n o}$.

C. you will starve yourself.

alternatively, you can bring some drinks.

it is not compulsory.

some snacks will be served there. 